\title{
Earth's 2006 encounter with comet 73P/Schwassmann-Wachmann: Products of nucleus fragmentation seen in closeup
}

\author{
Zdeněk Sekanina \\ Jet Propulsion Laboratory, California Institute of Technology, Pasadena, CA 91109, USA \\ email: zs@sek.jpl.nasa.gov
}

\begin{abstract}
The large numbers of nucleus fragments observed are a spectacular illustration of the process of cascading fragmentation in progress, a concept introduced to interpret the properties of the Kreutz system of sungrazers and comet D/1993 F2. The objective is to describe the fragmentation sequence and hierarchy of comet $73 \mathrm{P}$, the nature of the fragmentation process and observed events, and the expected future evolution of this comet. The orbital arc populated by the fragments refers to an interval of 3.74 days in the perihelion time. This result suggests that they all could be products (but not necessarily first-generation fragments) of two 1995 events, in early September (involving an enormous outburst) and at the beginning of November. The interval of perihelion times is equivalent to a range of about $2.5 \mathrm{~m} / \mathrm{s}$ in separation velocity or 0.00012 the Sun's attraction in nongravitational deceleration. Their combined effect suggests minor orbital momentum changes acquired during fragmentation and decelerations compatible with survival over two revolutions about the Sun. Fragment B is a likely first-generation product of one of the 1995 events. From the behavior of the primary fragment C, 73P is not a dying comet, even though fragment B and others were episodically breaking up into many pieces. Each episode began with the sudden appearance of a starlike nucleus condensation and a rapidly expanding outburst, followed by a development of jets, and a gradual tailward extension of the fading condensation, until the discrete masses embedded in it could be resolved. In April-May, this debris traveled first to the southwest, but models show their eventual motion toward the projected orbit. Fainter fragments were imaged over limited time, apparently because of their erratic activity (interspersed with periods of dormancy) rather than improptu disintegration. A dust trail joining the fragments and reminiscent of comet 141P/Machholz suggests that cascading fragmentation exerts itself profoundly over an extremely broad mass range of particulate debris.
\end{abstract}

Keywords. Comet, fragmentation; data analysis

\section{Introduction}

Only since recently has it been recognized that fragmentation is an omnipresent process among comets that proceeds at all heliocentric distances. Fragmentation is also increasingly perceived as the dominant process of cometary demise, likely to account in most (though not necessarily all) cases for the end state.

Perhaps the most fascinating research opportunity that cometary fragmentation offers to a scientist is the benefit to examine, at no extra cost, the interior of the nucleus as subsurface areas suddenly become exposed to direct solar radiation and other outerspace effects. The fragmentation process itself is also of much interest, especially for comets that break up nontidally (Sekanina 1997), having experienced no close approach to the Sun or Jupiter in the past. Fragmentation of a comet's nucleus is facilitated by its extremely low mechanical strength (e.g., Whipple 1950, 1963, A'Hearn et al. 2005) and is probably also aided significantly by major variations in the mechanical-strength distribution throughout the nucleus interior. It appears that nontidal fragmentation is 


\section{CASCADING FRAGMENTATION OF COMETARY NUCLEI} (SCHEMATICALLY)

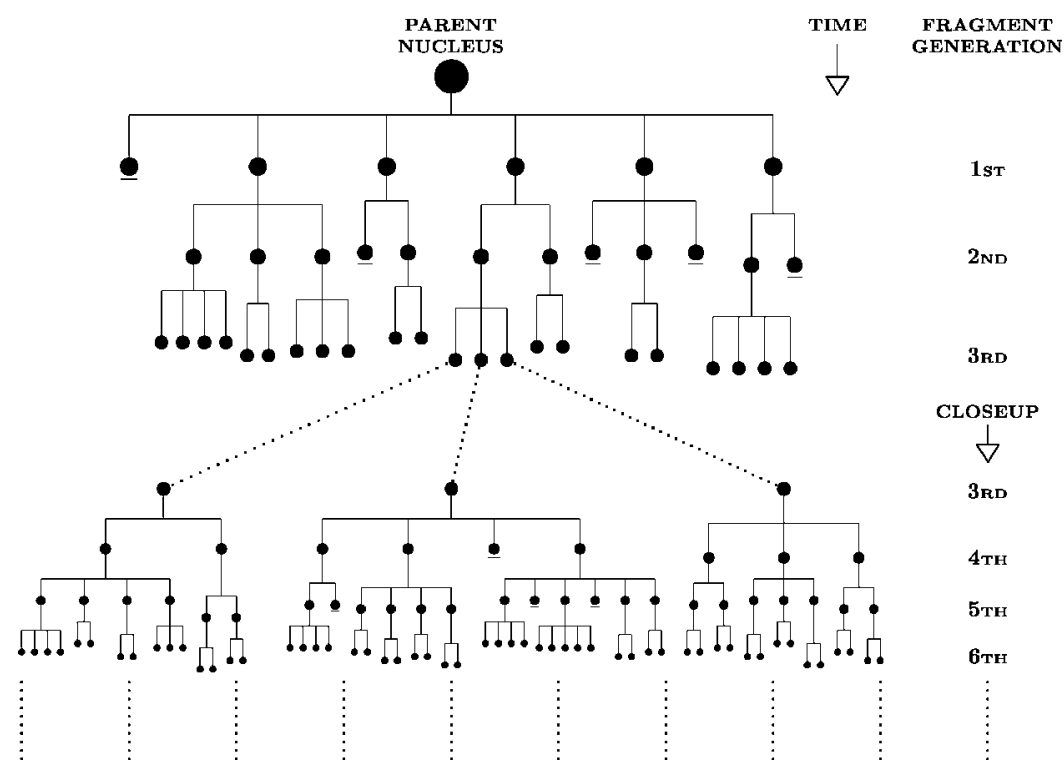

Figure 1. Schematic representation of the process of cascading fragmentation, proposed for cometary nuclei. The underscored symbols identify the surviving fragments whose (incomplete) disintegration has terminated. The first three generations of fragments are displayed in the upper part; a detail of the fourth, fifth, and sixth generations is in the lower part.

triggered by one or more of three possible mechanisms: rotational tension, thermal stress, and pressure of outflowing gases from discrete sources, especially when the volatiles are trapped beneath the surface.

Effects on split comets can be discriminated into two categories: nondestructive and cataclysmic. An event of the first category is survived by at least one fragment nearly unaffected, so that the comet's life goes on. By contrast, an event of the second category destroys the comet completely on a very short time scale. In this paper I offer some early results on nondestructive fragmentation of comet 73P/Schwassmann-Wachmann 3.

\section{Cascading Fragmentation}

In 1996, my interest in cometary fragmentation was aroused by the astonishingly high discovery rate of dwarf comets of the Kreutz sungrazer system, all of which, before reaching perihelion, fade and vanish while imaged with two coronagraphs onboard the NASA/ESA Solar and Heliospheric Observatory (SOHO). Following an earlier investigation of comet D/1993 F2 (Sekanina et al. 1998) that first split and eventually collided with Jupiter, I proposed a concept of cascading fragmentation (Sekanina 2002) to explain the observed sequence of events (Figure 1). In this scenario, the original parent comet continues to break up over and over again, with an ever larger number of fragments of ever smaller size being generated episodically.

In the case of the Kreutz system, the inevitability of this scenario has amply been documented by two facts: (i) all minor sungrazers, discovered coronagraphically (mostly with the SOHO instruments) fail to survive their perihelion passage, implying that their existence as separate objects cannot predate the previous perihelion passage and their parent bodies, in order to survive, must have been substantially (orders of magnitude) 


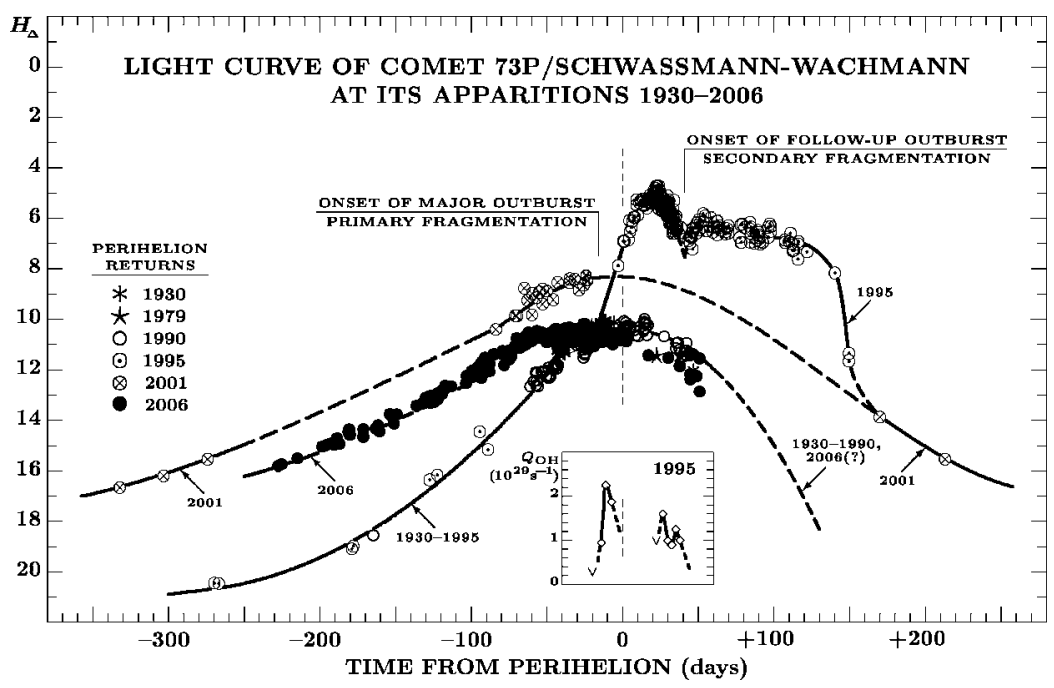

Figure 2. Light curve of comet 73P during the six apparitions 1930-2006. The plotted magnitude $H_{\Delta}$ was corrected for effects of geocentric distance and normalized to the visual system. The onset times of the two outbursts in 1995 and their apparent coincidence with the times of the primary and secondary events of nucleus fragmentation are depicted. During the 2001 and 2006 apparitions the magnitudes plotted refer to the primary fragment $\mathrm{C}$. The inset shows the parallel 1995 variations in the hydroxyl production rate, measured by Crovisier et al. (1996).

more massive; and (ii) the minor sungrazers have a strong tendency to arrive at the Sun in pairs or clusters, moving along trajectories that are similar but by no means identical. The differences indicate orbital perturbations caused by separation velocities of a few $\mathrm{m} / \mathrm{s}$ acquired during fragmentation events far from the Sun, where the orbital velocity does not exceed a few tens $\mathrm{m} / \mathrm{s}$.

In the work on comet D/1993 F2 (Sekanina et al. 1998), we were able to determine the family tree of the fragmentation products. By the time the comet collided with Jupiter two years after the initial, tidally-triggered breakup, fragments of the first, second, and third generations were identified. With many dozens of fragments now observed in the orbit of comet $73 \mathrm{P}$, a new opportunity is presented to test the concept of cascading fragmentation in a case where the initial breakup was nontidal in nature.

\section{Brief History of Comet 73P/Schwassmann-Wachmann}

This comet is a member of the Jupiter family of short-period comets, with an orbital period of 5.4 years and perihelion near 1 AU. The 2006 apparition is the comet's sixth observed return to the Sun. Its light-curve evolution, displayed in Figure 2, had been unexciting until early September 1995, when an enormous outburst began about two weeks before perihelion. The event was first detected by Crovisier et al. (1996) as an $\mathrm{OH}$ production increase at $18 \mathrm{~cm}$. The optical confirmation came several days later. Figure 2 shows that the first outburst was followed by a second, smaller one nearly two months later and that the comet's brightness remained elevated not only for the rest of the 1995 apparition, but also in 2001 and 2006.

The multiplicity of the nucleus was first detected by Boehnhardt \& Käufl (1995) at the European Southern Observatory (ESO) on December 12, more than three months after the onset of the first outburst. Three optically detected fragments were aligned in a nearly rectilinear chain about $4^{\prime \prime}$ long. According to the notation by Marsden (1996a), 
the westernmost fragment became known as $\mathrm{A}$, the easternmost as $\mathrm{C}$, and the middle, initially the faintest one, as B. A fourth fragment, called D, was reported independently by J. V. Scotti and A. Galád only on 1995 December 27-29 (Marsden 1996a).

Subsequent close inspection of the ESO images taken by K. Reinsch on November 28 and by J. Storm on December 2 revealed that the comet was already double (Boehnhardt et al. 1996). Nucleus C was clearly the primary (and presumably the most massive) fragment (Marsden 1996a). Fragments A, B, and C were observed until mid-February 1996. After conjunction with the Sun, in late August 1996, only C and one companion were detected and observed for more than three months (Marsden 1996b, 1997).

When the comet was recovered in November 2000, the primary and two companions were detected; one of them, officially designated E, appeared to be a new fragment (Green 2000). After perihelion, from July to December 2001, the primary and a single companion were under observation at ESO (Boehnhardt et al. 2002).

A recent effort to sort out the identity of the fragments and to establish their fragmentation sequence and hierarchy (Sekanina 2005) produced surprising results. All examined companions were found to have separated from the parent comet they shared with the main fragment $\mathrm{C}$ rather than from one another. Fragment A was short lived, seen only in the late 1995 and early 1996, separating in late October 1995 and moving rapidly away from C. Fragment E, introduced as a new fragment in 2000, turned out to be identical with the companion from the late 1996; it was found to have separated from the parent at the onset time of the major 1995 outburst. Fragment B could not satisfactorily be linked with the 2001 companion, nor with the other 2000 companion. These two could, however, be linked together and identified as a new fragment $\mathrm{F}$, which separated from what was left of the parent nucleus at the onset time of the follow-up outburst (see Figure 2). It was concluded that fragment B was observed only in the late 1995 and early 1996 and that it separated probably during the major outburst. Thus, a strong correlation has been established between the fragmentation events and the outbursts. The 2006 ephemerides for companions E, F, and B were calculated, with the proviso that the ephemeris for B was very uncertain because of severe extrapolation.

\section{Current Return of Comet 73P: A String of Nucleus Fragments}

The main comet (fragment C) was recovered by C. Hergenrother with 1.2-meter reflector at Mount Hopkins on 2005 October 22 (Green 2005), 227 days before perihelion. The light curve available at the time of this writing (the beginning of August 2006) shows that along much of the preperihelion arc of the orbit this surviving fragment was still brighter than the parent comet in 1930-1995 but that near and several weeks after perihelion the light curve of $\mathrm{C}$ was running at or slightly below that of the parent (Figure 2).

The first companion to $73 \mathrm{P}$ during the current return was discovered by J. A. Farrell with his 0.41-meter reflector on 2006 January 6, or 151 days before perihelion of fragment C; I tentatively identified it with fragment B from 1995-1996 (Green 2006a). Next came R. A. Tucker's and E. J. Christensen's independent discoveries of fragment G on 2006 February 20-24 (Green 2006b). Figure 3 indicates that large numbers of additional fragments were discovered starting from March 4, most of them with the 1.5-meter reflector at Mount Lemmon (Green 2006c). An official count - 65 including fragment $\mathrm{C}$ - is incomplete, because none of the several dozen minifragments seen in the images of companions B and G taken with the Hubble Space Telescope (HST) on April 18-20 has been accounted for. Similarly, the high-resolution images taken with the Very Large

$\dagger$ http://hubblesite.org/newscenter/newsdesk/archive/releases/2006/18 


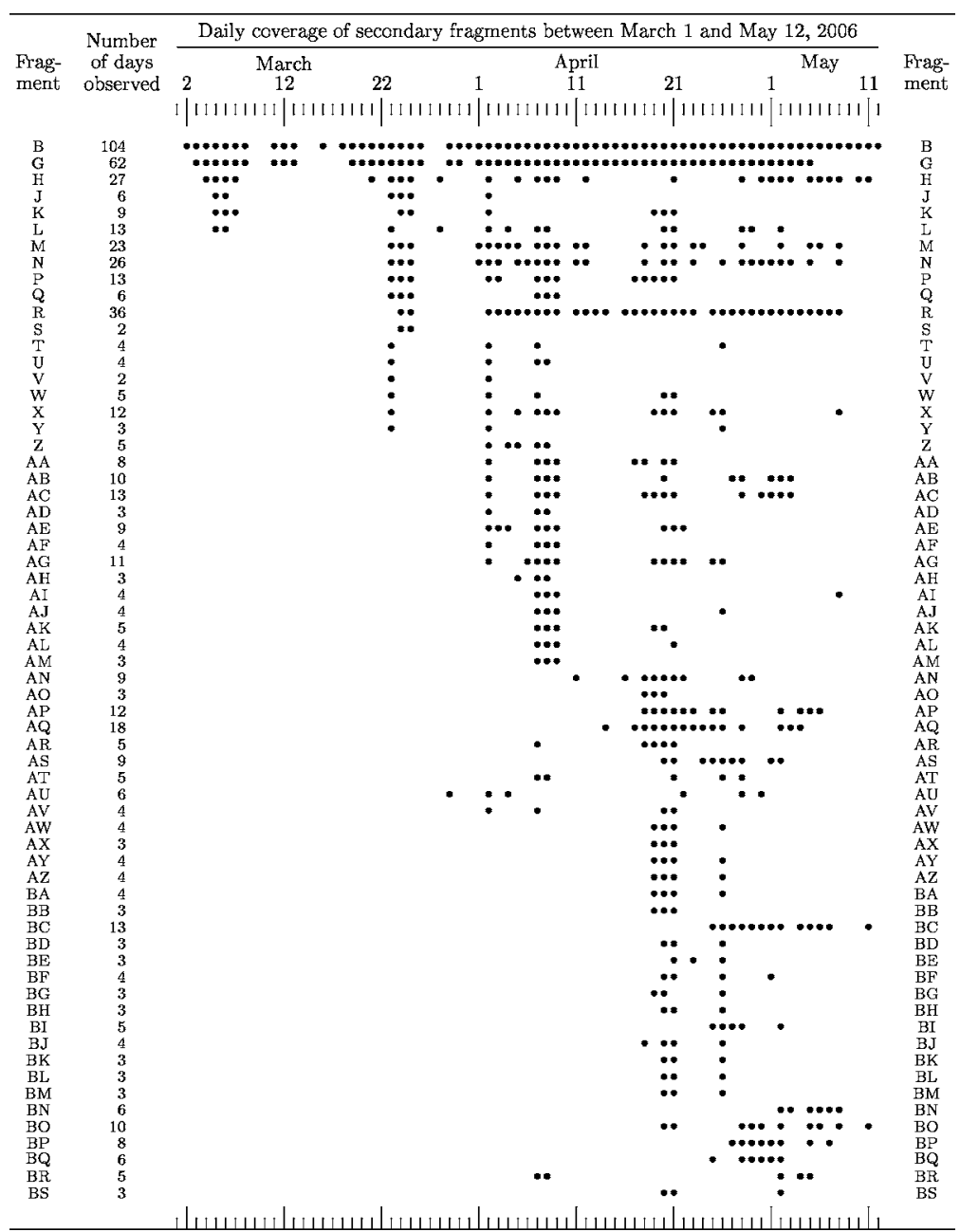

Figure 3. Astrometric observations of fragments of 73P between March 1 and May 12, 2006.

Telescope (Kueyen) at ESO's Cerro Paranal Station on April 23/24 depicted seven faint fragments closely trailing companion B $\ddagger$, while C. Hergenrother $\uparrow$ and others noticed short-lived fragments near B and $\mathrm{G}$ on numerous occasions in April and May. Additional minor fragments briefly accompanying or involved with the condensations $H, M, N, R$, etc. were also reported by observers from time to time. All the minifragments imaged near B have in the official count been "represented" by a single generic fragment AQ, whereas those near $\mathrm{G}$ and other companions have mostly been ignored.

Figure 4 compares the light curves of three fragments. It is noticed that the light curve of the main fragment $\mathrm{C}$ is very smooth, while that of $\mathrm{B}$, the second brightest fragment, has a distinctly steeper slope with three outbursts before perihelion. The entire light curve of $\mathrm{N}$, one of the fainter companions, consists entirely of rapid fluctuations. It seems that the fainter (and, presumably, the smaller and less massive) the fragment is, the more erratic its activity, which apparently implies the object's lesser textural homogeneity and mechanical stability, and therefore its shorter lifetime.

\section{$\ddagger$ http://www.eso.org/outreach/press-rel/pr-2006/pr-15-06.html \\ I http://www.lpl.arizona.edu/ chergen/73P.html}




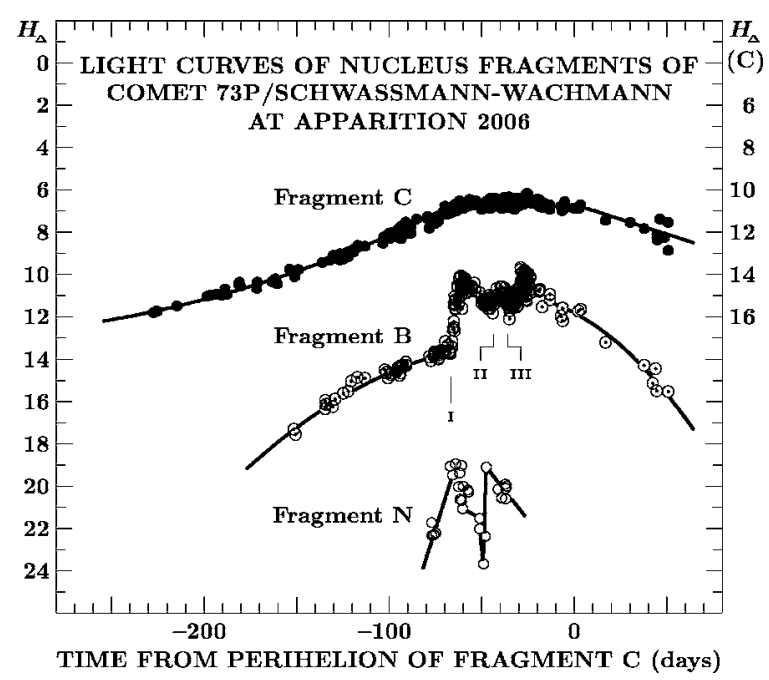

Figure 4. Light curves of three fragments of comet 73P in 2006. The plotted magnitude $H_{\Delta}$ was normalized to $1 \mathrm{AU}$ and to the visual system. The smoothness of the curve for fragment C contrasts with the three outbursts of fragment B, marked as I (with the onset on April 1), II (April 24), and III (May 2), and with the ragged curve of fragment N. The light curve for the primary fragment $\mathrm{C}$ was moved 4 magnitudes up (right scale) to avoid a congestion of the curves for B and C. At the peak of outbursts I and III, fragment B was slightly brighter than C.

A fragmentation episode, like those experienced by companion B, begins with the sudden appearance of a starlike nucleus condensation and an outburst, followed by a development of jets and by a gradual tailward extension of the fading condensation, until the discrete, boulder-sized masses (minifragments) embedded in it are resolved. The described phenomena are products of a rapidly expanding cloud of microscopic dust that makes up the lower end of the size spectrum of the released debris. A procession of minifragments was observed to follow each of the outbursts of companion B as well as the flare-ups of numerous other companions.

Figure 5 presents simple fragmentation models (Sec. 5 ), which show a range of possible scenarios for the April-May time frame. It is noted that in this period of time, freshly released fragments traveled, relative to their parent, first to the southwest and only later to the north, toward the projected orbit. On the other hand, very old fragments traveled essentially along the orbit, explaining the observed string of lined-up fragments.

The motions of these fragments are crudely described by the orbit of $\mathrm{C}$ with shifted perihelion times. Figure 6 shows a peculiar effect: the expected perihelion times of the two companions from 2000/2001 coincide with the gaps in the histogram. Is this telling us that $\mathrm{E}$ and $\mathrm{F}$ have disintegrated into the observed populations of fragments? Their majority should indeed pass through perihelion at slightly later times. The range of perihelion times of the examined fragments, 3.74 days, is equivalent to an orbital-velocity increment of about $2.5 \mathrm{~m} / \mathrm{s}$ or to a differential deceleration of 0.00012 the Sun's gravitational acceleration for a single event having occurred in September-November 1995. These values suggest that as few as 1-2 episodes per fragment would suffice to explain the entire span of perihelion times.

The complexities of the spatial distribution of fragments are illustrated by their four subsets on four dates in Figures 7-10. The apparent resiliency of a number of fragments and the fact that fragment $\mathrm{C}$ continues to be in good health suggest that $73 \mathrm{P}$ is not yet a dying comet, contrary to recently expressed opinions in some magazines. 


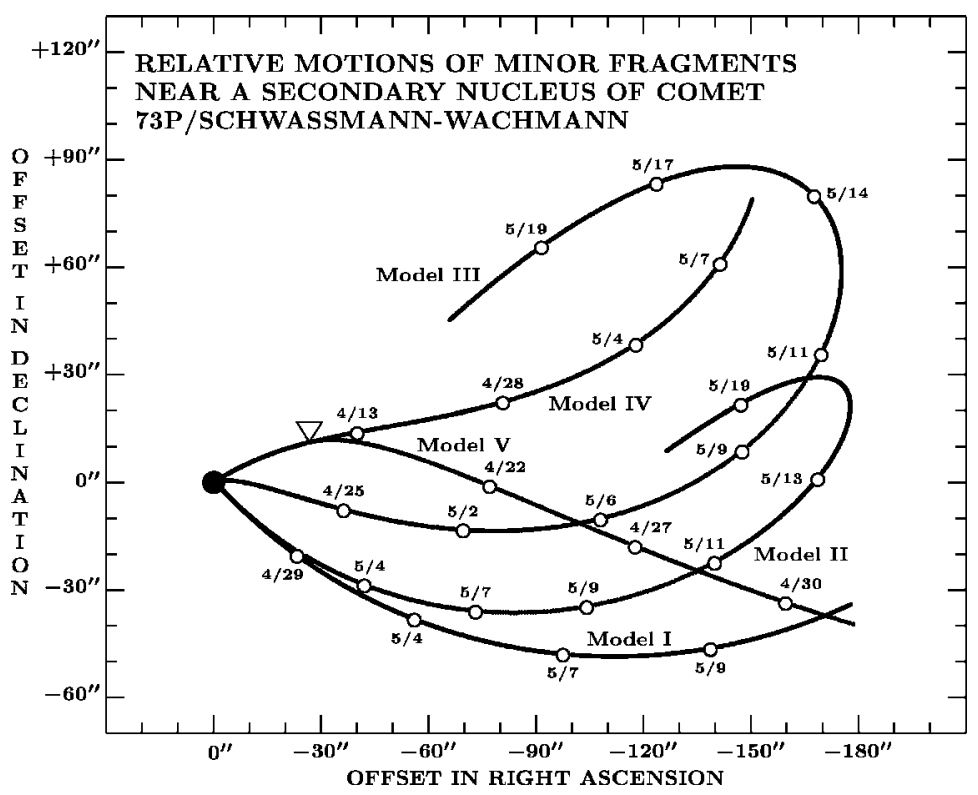

Figure 5. Models for relative motions of higher-generation fragments in the proximity of a first-generation (reference) fragment of 73P during April and May 2006. Times of separation from the reference fragment and decelerations (in units of $10^{-5}$ the Sun's gravitational acceleration): 2006 Apr 1 and 40 units for Model I; 2006 Apr 1 and 30 units for Model II; 2006 Jan 1 and 6 units for Model III; 2001 Jan 1 and 0.02 units for Model IV; in Model V, the fragment separated from Model IV fragment (rather than from the reference fragment) on 2006 Apr 1 at a location marked with a triangle, with a deceleration of 60 units.

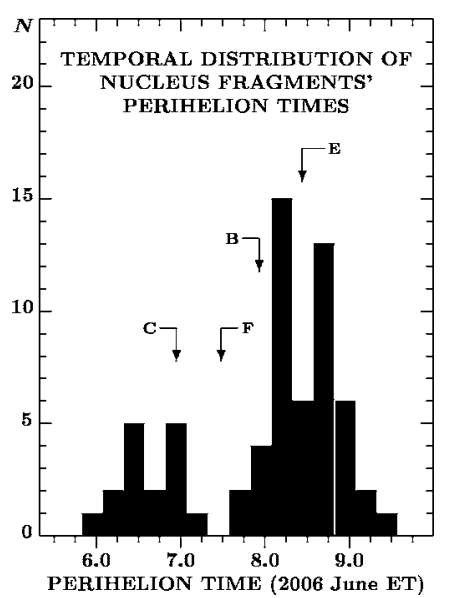

Figure 6. Histogram of the temporal distribution of perihelion times for 65 nucleus fragments of $73 \mathrm{P}$ with official designation. The times span an interval of 3.74 days. The times of the main fragment $\mathrm{C}$ and the brightest secondary fragment $\mathrm{B}$ and the predicted locations of fragments $\mathrm{E}$ and $\mathrm{F}$ (observed during the previous apparitions but not in 2006) are marked with the arrows. Note that the times for $\mathrm{E}$ and $\mathrm{F}$ match the gaps in the temporal distribution.

One of the products of the process of cascading fragmentation is the formation of a dust trail, the phenomenon investigated for a number of periodic comets (e.g., Sykes \& Walker 1992). The trail of coarse-grain and pebble-sized debris of $73 \mathrm{P}$ detected in the 


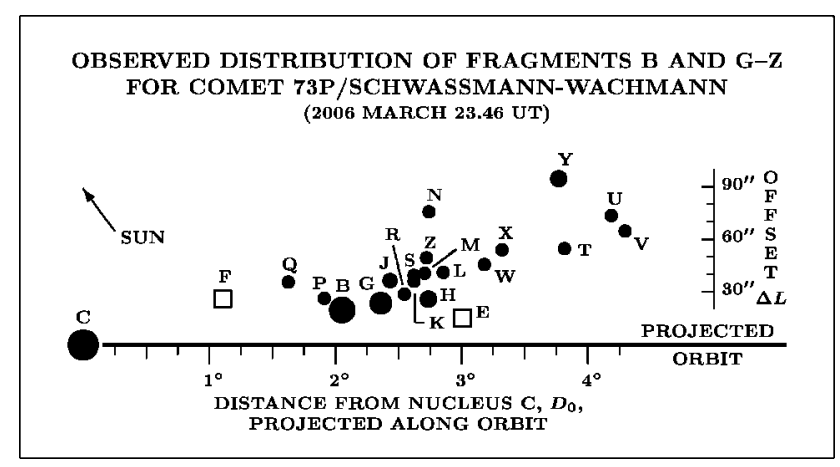

Figure 7. The locations of fragments $\mathrm{B}$ and $\mathrm{G}$ through $\mathrm{Z}$ relative to the main fragment $\mathrm{C}$ in projection onto the plane of the sky on 2006 March 23.46 UT. Offset $\Delta L$ is a displacement from the direction of the projected orbit behind fragment $\mathrm{C}$. The magnitude of this displacement is magnified by a factor of 50 relative to the scale along the orbit. The circle size corresponds approximately to the peak brightness reported. The squares are the predicted locations of fragments $\mathrm{E}$ and F. The direction to the Sun is also shown.

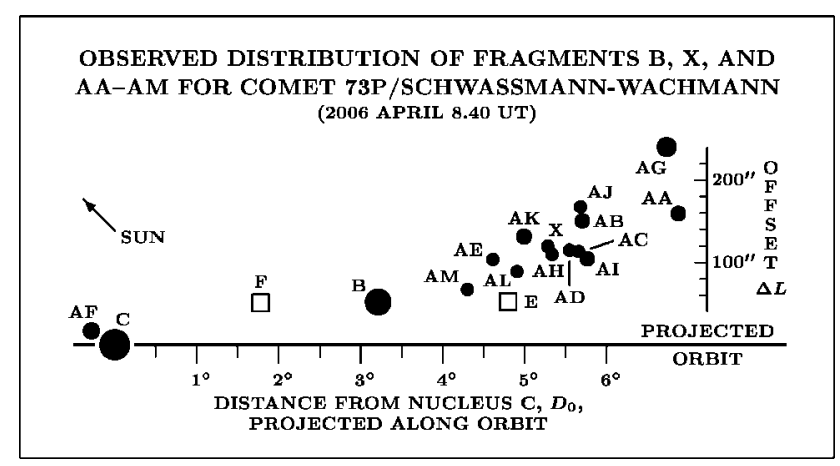

Figure 8. The locations of fragments AA-AM relative to fragment $\mathrm{C}$ in projection onto the plane of the sky on 2006 April 8.40 UT. Fragments B and X are plotted to allow comparison with Figure 7 . Offset $\Delta L$ is magnified by a factor of 36 relative to the scale along the orbit. For more information, see the caption to Figure 7.

infrared by the Spitzer Space Telescope $\dagger$ is apparently of the same nature as features observed in other split comets in the optical wavelengths, namely, a sheath of material in the sungrazer C/1882 R1 (e.g., Kreutz 1888) and a dust filament or trail in D/1993 F2 (e.g., Weaver et al. 1994) and 141P/Machholz (cf. Sekanina 1999).

\section{Analysis, Early Results, and Conclusions}

Only preliminary results are available at present, based on nearly 8000 astrometric positions between January 6 and the end of July 2006, extracted from the Minor Planet Electronic Circulars (MPECs) 2006-B20 through 2006-P18 published by the IAU Minor Planet Center. $\ddagger$ The same data are subsequently published, once a month, in the Minor Planet Circulars. There are currently over 1600 positions for fragment B, over 800 positions for G, over 400 for R, etc. The 2006 light-curve data are mostly from the web site of the International Comet Quarterly, $\mathbf{q}$ but some are from the MPECs.

$\dagger$ http://www.spitzer.caltech.edu/Media/releases/ssc2006-13

$\ddagger$ http://www.cfa.harvard.edu/mpec/RecentMPECs.html

I http://cfa-www.harvard.edu/icq/CometMags.html 


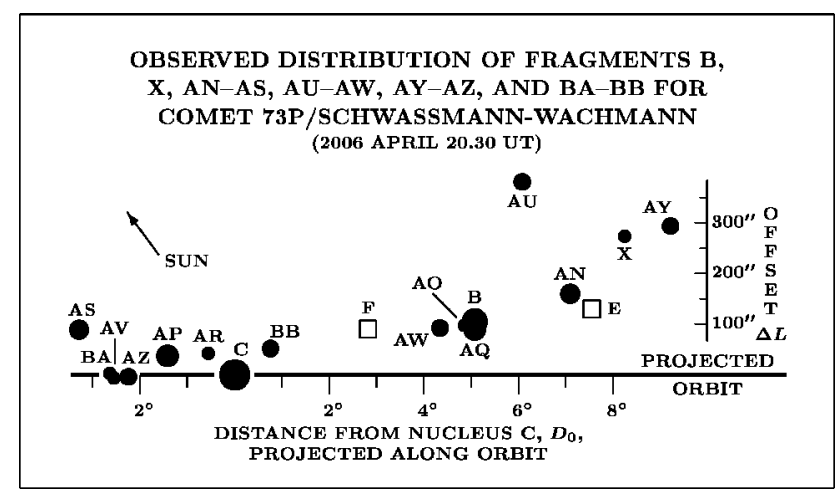

Figure 9. The locations of fragments AN-AS, AU-AW, AY-AZ, and BA-BB relative to fragment $\mathrm{C}$ in projection on the plane of the sky on 2006 April 20.30 UT. Fragments B and X are plotted to allow comparison with Figures 7 and 8 . Offset $\Delta L$ is magnified by a factor of 38.4 relative to the scale along the orbit. For more information, see the caption to Figure 7.

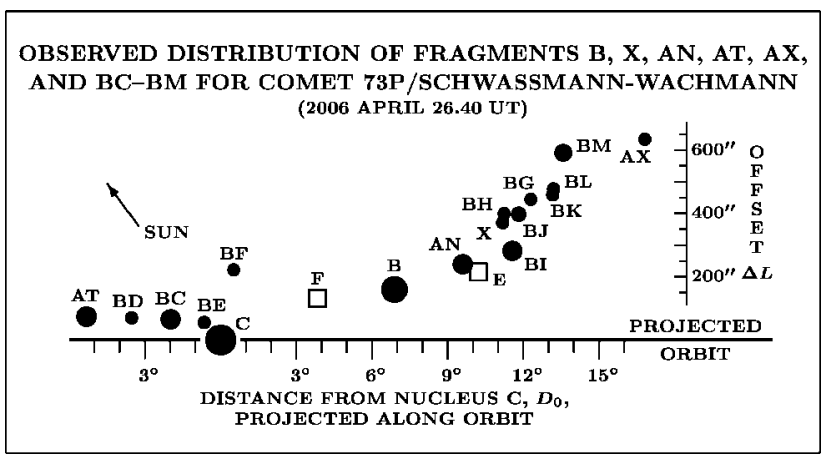

Figure 10. The locations of fragments AT, AX, and BC-MN relative to fragment $\mathrm{C}$ in projection onto the plane of the sky on 2006 April 26.40 UT. Fragments B, X, and AN are plotted to allow comparison with Figures 7-9. Offset $\Delta L$ is magnified by a factor of 45 . For more information, see the caption to Figure 7.

The extensively tested fragmentation model (Sekanina 1978, 1982) is used to fit the relative motion of fragments in each examined pair by employing their offsets in right ascension and declination. The model allows the user to solve for up to five parameters: the time of fragmentation; the radial, transverse, and normal components of the separation velocity (referred to the orbit plane of the shared parent and aligned with the Sun-comet direction); and the differential nongravitational deceleration. The procedure involves an iterative, least-squares, differential-correction algorithm that searches for an optimum fit. One can solve for any combination of fewer than the five parameters. Because of the long periods of time involved in the case of $73 \mathrm{P}$ (1995-2006), the differential planetary perturbations are accounted for in a code's version that I developed in a joint effort with P. W. Chodas. With 65 fragments there are more than 2000 pair combinations possible, although many can be ruled out as implausible. A poor distribution of residuals from a solution means that the fragments in the chosen pair do not share a common parent.

The separation velocity, which is particularly important for understanding the existence of fragments on the leading side of $\mathrm{C}$ (such as AT in Figure 10), is interpreted as an extra momentum acquired during breakup by the smaller fragment in a pair relative to the reference mass. In reality, of course, either fragment acquires a net orbital momentum change, albeit the one for the reference mass is much smaller. By the same 
token, if B had been a first-generation fragment before the three outbursts (Figure 4), it became a second-generation fragment after outburst I, a third-generation fragment after outburst II, and a fourth-generation fragment after outburst III, even though it was then still called B and its net orbital momentum changed hardly at all due to the three events.

The early results show that, as expected, companion B separated from C in 1995, most probably in early November, and that the cluster of minifragments near B observed with the HST on April 18-20 (Sec. 4) was released on about April 1 (outburst I in Figure 4), thus illustrating (with many other similar events) the process of cascading fragmentation in progress and confirming a correlation between outbursts and fragmentation. Finally, it has by now become clear that the process begun with the fragmentation events in 1995 has not been cataclysmic and that $73 \mathrm{P}$ shows at present no signs of being a dying comet.

\section{Acknowledgements}

This research was carried out at the Jet Propulsion Laboratory, California Institute of Technology, under a contract with the National Aeronautics and Space Administration.

\section{References}

A'Hearn, M. F. and the Depp Impact Team 2005, Science 310, 258

Boehnhardt, H., \& Käufl, H. U. 1995, IAU Circ. 6274

Boehnhardt, H., Holdstock, S., Hainaut, O., Tozzi, G. P., Benetti, S., \& Licandro, J. 2002, Earth Moon Plan. 90, 131

Boehnhardt, H., Käuf, H. U., Goudfrooij, P., Storm, J., Manfroid, J., \& Reinsch, K. 1996, ESO Messenger No. 84, p. 26

Crovisier, J., Bockelée-Morvan, D., Gérard, E., Rauer, H., Biver, N., Colom, P., \& Jorda, L. 1996, AA 310, L17

Green, D. W. E., ed. 2000, IAU Circ. 7534

Green, D. W. E., ed. 2005, IAU Circ. 8623

Green, D. W. E., ed. 2006a, IAU Circ. 8659, 8660

Green, D. W. E., ed. 2006b, IAU Circ. 8679

Green, D. W. E., ed. 2006c, IAU Circ. 8685, 8692, 8693, 8703, 8704, 8709, 8715

Kreutz, H. 1888, Publ. Kiel Sternw. No. 3, p. 1

Marsden, B. G., ed. 1996a, IAU Circ. 6301

Marsden, B. G., ed. 1996b, Minor Planet Circ. 28339

Marsden, B. G., ed. 1997, Minor Planet Circ. 28917

Sekanina, Z. 1978, Icarus 33, 173

Sekanina, Z. 1982, in: L. L. Wilkening (ed.), Comets (Tucson: Univ. Arizona), p. 251

Sekanina, Z. 1997, AA 318, L5

Sekanina, Z. 1999, AA 342, 285

Sekanina, Z. 2002, ApJ 566, 577

Sekanina, Z. 2005, Internat. Comet Quart. 27, 225

Sekanina, Z., Chodas, P. W., \& Yeomans, D. K. 1998, Planet. Space Sci. 46, 21

Sykes, M. V. \& Walker, R. G. 1992, Icarus 95, 180

Weaver, H. A., Feldman, P. D., A'Hearn, M. F., Arpigny, C., Brown, R. A., Helin, E. F., Levy, D. H., Marsden, B. G., Meech, K. J., Larson, S. M., Noll, K. S., Scotti, J. V., Sekanina, Z., Shoemaker, C. S., Shoemaker, E. M., Smith, T. E., Storrs, A. D., Yeomans, D. K., \& Zellner, B. 1994, Science 263, 787

Whipple, F. L. 1950, ApJ 111, 375

Whipple, F. L. 1963 in: B. M. Middlehurst \& G. P. Kuiper (eds.), The Moon, Meteorites, and Comets (Chicago: Univ. Chicago), p. 639 